\title{
AÇÃO DE DERIVADOS DO ÁCIDO BETULÍNICO EM CÉLULAS DE CÂNCER DE CÓRTEX ADRENAL
}

\author{
ACTION OF BETULINIC ACID DERIVATIVES IN ADRENAL CORTEX \\ CANCER CELL LINES
}

Margot Marie Martin1; Adriana Aya Yamaguchi2; Paloma Sabrina Ribeiro2; Bonald Cavalcante Figueiredo2; Sandro José Ribeiro Bonatto2

\section{RESUMO}

Fundamento: $O$ câncer de córtex de adrenal (CCA) é um câncer geralmente pediátrico que tem incidência relativamente baixa nos Estados Unidos, de 0,3 a cada milhão de crianças com idade menor do que 15 anos. Entretanto, este tumor, na região Sul do Brasil, tem uma incidência maior do que em outras áreas. Ainda é um tumor com prognostico desfavorável. Seu tratamento é cirúrgico e tem como quimioterapia adjuvante, o mitotano. 0 ácido betulínico é um composto natural descrito na literatura como potente agente citotóxico com índice terapêutico favorável. Objetivo: Este trabalho teve como intuito investigar o efeito de derivados do ácido betulínico em linhagens de H295R de CCA. Métodos: Foi avaliada a viabilidade celular de H295R quando exposta aos derivados de ácido betulínico DAB2 e DAB3 e comparada a de um grupo controle que não foi exposto a esses compostos. Numa segunda etapa do experimento, foi avaliada a viabilidade celular de fibroblastos, para estimar a seletividade dos derivados. Resultados: A viabilidade celular de H295R diminuiu significativamente nas diluições de DAB2 1:1000 e nas diluições 1:5000 de DAB3. Nenhum dos compostos diminuiu significativamente a viabilidade dos fibroblastos. Conclusão: Os dados do presente estudo apontaram que os derivados de ácido betulínico DAB2 e DAB3 têm potencial para desenvolvimento de uma droga citotóxica para o CCA com índice terapêutico favorável.

Descritores: carcinoma adrenal, ácido betulínico, infância

\section{ABSTRACT}

Background: Adrenocortical Carcinoma (ACC) is a pediatric cancer that has relatively low incidence in the United States, 0.3 per million children aged less than 15 years. In southern Brazil, however, this tumor has a higher incidence than in other areas.It is still a tumor with unfavorable prognosis. The primary treatment is surgical with mitotane as adjuvant chemotherapy. Betulinic acid is a natural compound described in the literature as a potent cytotoxic agent with a favorable therapeutic index.Objective: To investigate the effect of betulinic acid derivatives in H295R lines of CCA.Method: H295R cell when exposed to the derivatives DAB2 and DAB3 was assessed and compared to a control group that was not exposed to these compounds. In the second stage of the experiment, cell viability of fibroblasts was measured to estimate the selectivity of the derivatives.

Results: Cell viability decreased significantly in H295R at DAB2 dilutions of 1: 1000 and at DAB3 the dilutions of 1: 5000 .

Neither compound decreased significantly viability of fibroblasts.Conclusion: This study suggests that betulinic acid derivatives DAB2 and DAB3 have the potential for the development of a cytotoxic drug for CCA with a favorable therapeutic index.

Keywords: adrenocortical carcinoma, betulinic acid, childhood.

1- Aluno do Curso de Medicina da Pontifícia Universidade Católica do Paraná - PUCPR

2- Vinculados ao Instituto de Pesquisa Pelé Pequeno Príncipe 


\section{INTRODUÇÃO}

No Brasil, o câncer é a segunda causa de óbito em jovens com idade entre 1 e 19 anos, correspondendo a $8 \%$ de todas as mortes registradas em 20051. Essa doença se caracteriza por uma proliferação excessiva e não regulada de células modificadas, distintas das linhagens celulares que estão em sua origem. Pode resultar de alterações em qualquer tipo celular, podendo atingir uma grande diversidade de órgãos ${ }^{2}$. 0 câncer de córtex de adrenal (CCA) tem uma incidência nos Estados Unidos de 0,3 a cada milhão de crianças com idade menor do que 15 anos. Entretanto, este tumor, na região Sul do Brasil, tem uma incidência 10 a 15 vezes maior do que em outras áreas ${ }^{3}$. Essa discrepância pode advir de alterações genéticas nos habitantes dessas áreas. Modificações genéticas são divididas em anormalidades cromossômicas ou alterações em sequências normais dos nucleotídeos. Por isso, o câncer é qualificado como uma patologia em que há expressão gênica anormal2,4. Foi esclarecido que na região sul a maioria dos CCA de crianças tem uma origem semelhante, associada com a mutação TP53 $\mathrm{R} 337 \mathrm{H}$ encontrada na linhagem germinativa associada com perda do segundo alelo ${ }^{3}$. Estudos demonstraram que aproximadamente uma em cada 10 pessoas portadoras desta mutação desenvolverá este tipo de tumor5.

Devido à baixa incidência do CCA e da falta de estudos experimentais randomizados, a determinação da melhor abordagem terapêutica é um desafio. A ressecção cirúrgica do carcinoma é o tratamento de escolha para os estágios I e II da doença. Enquanto para pacientes em estágios III e IV da doença, é escolhida uma terapia adjuvante com mitotano, além da cirurgia. Pacientes diagnosticados numa fase mais precoce têm uma melhora da sobrevida de 5 anos. No entanto, os benefícios clínicos do uso do mitotano são questionáveis 6.0 óbito precoce é atribuído mais à toxicidade do tratamento, do que à progressão do câncer ${ }^{7}$.Assim, é necessária a prospecção de novos compostos que possam estabelecer tratamentos menos nocivos e mais eficazes.

Nesse contexto, plantas são uma fonte para descoberta de novas substâncias com ação citotóxica e antitumoral contra várias linhagens de neoplasias 8.0 ácido betulínico é um triterpeno pentacíclico encontrado em várias plantas como Ziziphus spp.; Syzygium spp.; Diospyros spp. e Paeonia spp. Este composto é descrito na literatura como potencial substância anti-inflamatória, antibacteriana, analgésica e antineoplásica. Sua ação citotóxica contra várias linhagens tumorais foi comprovada e, leva ao aumento da permeabilidade mitocondrial com ativação das caspases e a fragmentação nuclear9. A vantagem oferecida por esta substância é a seletividade que ela oferece, diminuindo riscos de efeitos colaterais sistêmicos, e seu largo espectro de mecanismos de ação que sugerem uma ação contra células resistentes a outros agentes terapêuticos por não ter como alvo o p5310,11. Do ácido betulínico, que passa por diversos processos químicos, são extraídos derivados para tentar alcançar a otimização de sua já estabelecida ação citotóxica9 ${ }^{\text {. Shi et }}$ al. descreveram que através da alteração da estrutura química é possível aprimorar o seu efeito em oito a nove vezes ${ }^{12}$. Assim, derivados 2,4-DNPH (2,4dinitrophenylhydrazone) do ácido betulínico foram sintetizados, como o ácido 3-[(2,4-dinitrofenil)hidrazono]29-oxolupan-28-oico (DAB3) e o ácido 3-[(2,4-dinitrofenil) hidrazono]lup-20(29)-en-28-oico (DAB2) ${ }^{13}$.

A hipótese levantada foi que a proliferação das células tumorais é diminuída quando exposta a derivados do ácido betulínico DAB2 e DAB3. A presente proposta teve por objetivo principal avaliar o potencial efeito terapêutico antitumoral de derivados do ácido betulínico, extraído da Betula platyphylla, em linhagens de células de CCA, in vitro. Os objetivos secundários foram: a) Confirmar a ação citotóxica do ácido betulínico e de seus derivados em linhagens de H295-R; b) Quantificar este efeito nas células tumorais; (c) Avaliar se essa ação citotóxica está presente contra fibroblasto.

\section{METODOLOGIA}

Os experimentos foram realizados no Instituto de Pesquisa Pelé Pequeno Príncipe no ano de 2014 num quadro de iniciação cientifica.

Células da linhagem humana H295R de tumor de córtex adrenal foram mantidas em meio DMEM (Meio de Eagle modificado por Dulbecco) suplementado com $10 \%$ de soro fetal bovino. Para o teste do efeito antineoplásico dos derivados do ácido betulínico (DAB 2 e DAB 3) utilizouse ensaio clonogênico, no qual a viabilidade das células tumorais foi testada nas seguintes diluições: 1:10, 1:50, 1:100, 1:500, 1:1000, 1:5000 e 1:10000 sextuplicadas e comparada com a viabilidade de amostras controle de células não expostas a esta substancia. Nestes ensaios, foi avaliada a viabilidade desta linhagem celular em solução de MTT [3-(4,5-dimetiltiazol-2-il)-2,5-difeniltetrazolium brometo] em placas de 96 poços (volume final de $200 \mu \mathrm{L}$ ), a $37{ }^{\circ} \mathrm{C}$ em atmosfera de $95 \%$ ar / $05 \% \mathrm{CO} 2$, por 48 horas, com posterior leitura em espectrofotômetro de placa. Os valores sendo expressos em Abs/106 cel/ml. Este mesmo processo foi repetido 2 vezes.

Num segundo momento da pesquisa, fibroblastos de origem humana foram mantidos em meio DMEM (Meio de Eagle modificado por Dulbecco) suplementado com $10 \%$ de soro fetal bovino. Para o teste do efeito citotóxico em células constitutivas humanas dos derivados do ácido betulínico (DAB2 e DAB3) utilizou-se ensaio clonogênico, no qual as viabilidades dos fibroblastos foram testadas nas seguintes diluições: 1:10, 1:50, 1:100, 1:500, 1:1000, 1:5000 e 1:10000 e comparada com a viabilidade de amostras controle de células não expostas a esta substância. Nestes ensaios, foi avaliada a capacidade de proliferação desta linhagem celular em solução de MTT em placas de 96 poços (volume final de $200 \mu \mathrm{L}$ ), a $37{ }^{\circ} \mathrm{C}$ em atmosfera de $95 \%$ ar / 05\% CO2, por 48 horas, com posterior leitura em espectrofotômetro de placa. Os valores sendo expressos em Abs/106 cel/ml. Este mesmo processo foi repetido 3 vezes. 
Os valores determinados das viabilidades celulares, das duas etapas da pesquisa, foram interpretados com o auxílio do software GraphPad Prism ${ }^{\circledR}$ (GraphPad Prism versão 5.00 para Windows, GraphPad Software, San Diego California EUA) e por este transposto em gráficos. Os dados foram expressos como média \pm erro padrão da média (EPM) dos grupos experimentais e submetidos à análise de variância de uma via com o pósteste de Bonferroni para populações com distribuição gaussiana. Para populações que não seguiram distribuições gaussianas, foi utilizada análise de variância não-paramétrica (teste de Kruskal-Wallis), seguido de pósteste de Dunn. Teste " $t$ " de Student foi utilizado quando indicado. $p<0,05$ foi usado para indicar significância estatística.

\section{RESULTADOS}

Os resultados da viabilidade celular apresentada pela linhagem H295R utilizando o DAB3 foram as seguintes: no grupo controle foi de 0,9261 $\pm 0,08467$; nas diluições $1: 10$ foi $0,8562 \pm 0,07195,1: 50$ foi $0,7745 \pm$ $0,06738,1: 100$ foi $0,7791 \pm 0,08064,1: 500$ foi $0,7266 \pm$ $0,06509,1: 1.000$ foi $0,7015 \pm 0,05838,1: 5.000$ foi $0,6326 \pm 0,06631$ e $1: 10.000$ foi de $0,7176 \pm 0,06313$. A viabilidade do H295R diminuiu significativamente $(p<0,05)$ nas diluições de DAB3 1:5000, conforme observado no gráfico 1.

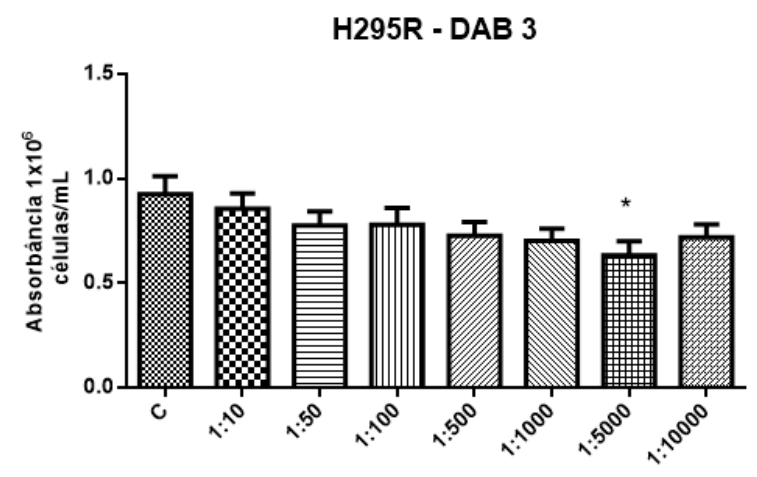

Gráfico 1: Viabilidade da linhagem H295-R de CCA, com grupo controle e as diferentes concentrações testadas de DAB3 por 48 horas. Os dados estão apresentados como médiatEPM de 2 experimentos em sextuplicatas. *viabilidade diminuída significativamente $(p<0,05)$ para 1:5000.

Nos grupos em que se utilizou DAB2 nas linhagens de CCA os resultados observados, descritos no gráfico 2 (em anexo), foram: o grupo controle apresentou proliferação celular de 0,9261 $\pm 0,08467$; enquanto nas diluições foram de $0,8222 \pm 0,06264$ para 1:10, $0,9122 \pm 0,1042$ para $1: 50,0,7277 \pm 0,07002$ para $1: 100$, $0,7741 \pm 0,07530$ para $1: 500,0,6928 \pm 0,06081$ para $1: 1000,0,7506 \pm 0,09106$ para $1: 5000,0,7660 \pm$ 0,07824 para 1:10000. A viabilidade diminuiu significativamente $(p<0,05)$ nas diluições de DAB2 1:1000, conforme destacado no gráfico 2 .
Conforme demonstrado no gráfico 3 , os resultados da viabilidade celular apresentada pelos fibroblastos utilizando o DAB3 foram as seguintes: no grupo controle foi de 0,4562 $\pm 0,04560$; nas diluições 1:10 foi $0,4347 \pm 0,03896,1: 50$ foi $0,4208 \pm 0,04338,1: 100$ foi $0,4264 \pm 0,04333,1: 500$ foi $0,4619 \pm 0,04610$, $1: 1.000$ foi $0,4313 \pm 0,04243,1: 5.000$ foi $0,4569 \pm$ 0,04772 e 1:10.000 foi de 0,3427 $\pm 0,01481$. O DAB3 não acometeu significativamente a viabilidade dos fibroblastos em nenhuma concentração.

Nos grupos em que se utilizou DAB2 em fibroblastos, os resultados observados descritos no gráfico 4 foram: o grupo controle apresentou proliferação celular de 0,4562 $\pm 0,04560$; enquanto nas diluições foram de $0,4388 \pm 0,02899$ para $1: 10,0,4269 \pm 0,03296$ para $1: 50$, $0,4760 \pm 0,04379$ para $1: 100,0,4406 \pm 0,03797$ para $1: 500,0,4636 \pm 0,03939$ para 1:1000, 0,4690 0,03966 para $1: 5000,0,4637 \pm 0,03776$ para $1: 10000$. A viabilidade não diminuiu significativamente em nenhuma das diluições de DAB2 conforme gráfico 4.

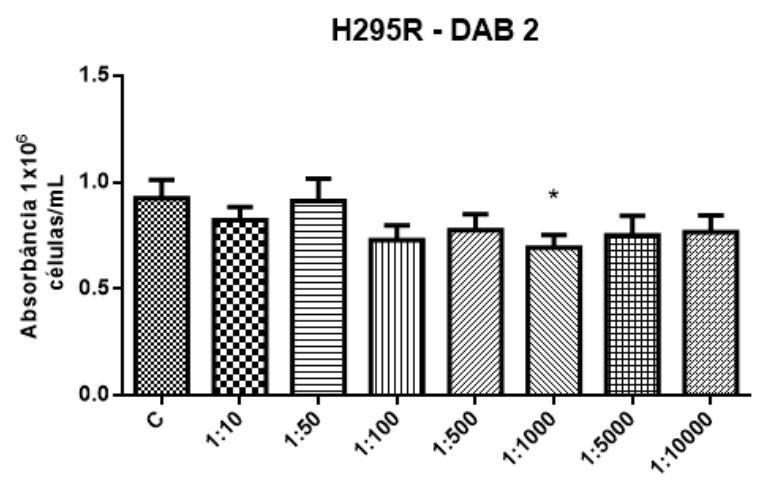

Gráfico 2: Viabilidade da linhagem H295-R de CCA, com grupo controle e as diferentes concentrações testadas de DAB2 por 48 horas. Os dados estão apresentados como médiatEPM de 2 experimentos em sextuplicatas. * viabilidade diminuída significativamente $(p<0,05)$ para 1:1000

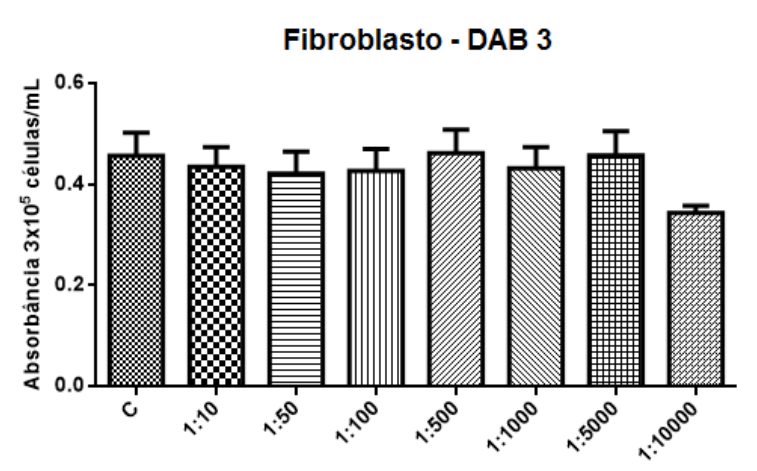

Gráfico 3: Viabilidade de fibroblastos, com grupo controle e as diferentes concentrações testadas de DAB3 por 48 horas. Os dados estão apresentados como médiatEPM de 3 experimentos em sextuplicatas. 
Fibroblasto - DAB2

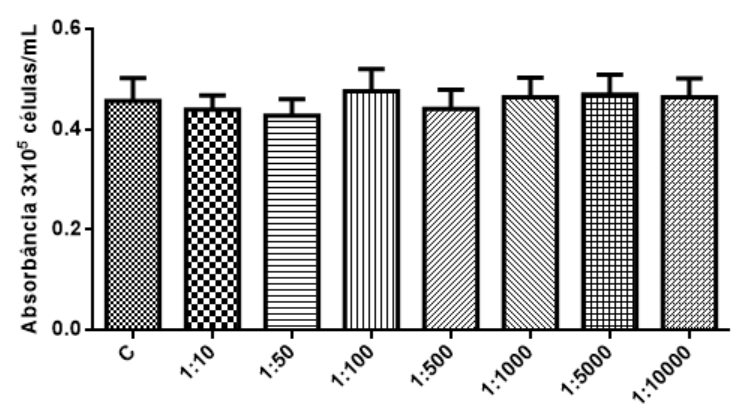

Gráfico 4: Viabilidade de fibroblastos, com grupo controle e as diferentes concentrações testadas de DAB2 por 48 horas. Os dados estão apresentados como médiatEPM de 3 experimentos em sextuplicatas.

\section{DISCUSSÃO}

Atualmente, o carcinoma de adrenal ainda tem um prognóstico desfavorável. Há uma real necessidade de aprimorar o tratamento oferecido. Pesquisas descrevem uma sobrevida em 5 anos dos pacientes com CCA que varia de 16 a 44\%. Essa diferença de sobrevida é explicada pelo prognóstico variável que depende principalmente do estágio do tumor e outras características como tamanho do tumor, taxas mitóticas e mutações do P53, mas ainda são poucas as características identificadas para estabelecer um prognóstico certo14. A única terapia que aumenta significativamente a sobrevida é a ressecção cirúrgica do tumor, particularmente quando detectado em fase I e II. Segundo Ng e Libertino, a sobrevivência média em pacientes com tumores irressecáveis é de 3 a 9 meses, enquanto os que puderam realizar ressecção completa tiveram uma sobrevida média de 13 a 28 meses ${ }^{15}$.

O mau prognóstico que apresenta este câncer é o que inspira a procura de novos compostos para alterar este quadro adverso. Estes podem ser procurados em produtos naturais já que mais da metade dos fármacos antitumorais que foram desenvolvidos nas últimas décadas se originaram destas substâncias, como a vincristina e o paclitaxel16,8. Tendo em vista o objetivo deste estudo, constatou-se diminuição da viabilidade de células neoplásicas de linhagens de câncer de adrenal quando expostas ao DAB2 e DAB3 em concentrações de 1:1000 e 1:5000, respectivamente. As outras concentrações, apesar de não expressarem valores significativos sugerem uma discreta diminuição dos valores. O ácido betulínico, que deu origem a esses derivados, já é descrito como forte candidato para estudos pré-clinicos e clínicos por ter citotoxicidade demonstrada em uma variedade ampla de linhagens tumorais ${ }^{10,17,18}$.

A indução a apoptose por via mitocondrial que culmina com ativação de caspases é um dos diversos mecanismos de ação executados pelo ácido betulínico que sugere que tenha ação contra células resistentes a outros agentes quimioterapêuticos ${ }^{2}$. Outros estudos demonstraram efeito antitumoral do ácido betulínico em melanomas, por ativação do p38 e de MAP cinases ${ }^{11}$. Ainda, poderia possuir outros efeitos como modulação do Fator de Crescimento do Endotélio Vascular (VEGF) e de proteínas inibidoras de apoptose (IAP) em células de câncer de próstata. Nesta mesma linhagem celular e ainda em câncer de cólon, descreveu-se ação do ácido betulínico em fatores de transcrições SP1, SP3 e SP417,18. Os resultados obtidos nos experimentos do trabalho presente corroboram com dados presentes na literatura que indicam que modificações na estrutura do ácido betulínico (AB) são capazes de produzir novos derivados potentes, os quais podem melhorar o perfil de toxicidade $e$ seletividade ${ }^{9,18}$. Segundo Baratto, num estudo que avaliou a citotoxicidade in vitro do $A B$ e derivados em cinco linhagens celulares neoplásicas, DAB3 apresentou resultados significativos em todas as linhagens testadas: exibiu ação em células de câncer de ovário, tireoide, melanoma, mama e pulmão. Enquanto DAB2 teve resultados significativos em somente 3 das linhagens: células de câncer de tireoide, mama e pulmão13.

A morbidade decorrente dos quimioterápicos ainda é uma limitação terapêutica19. Por isso, a inibição seletiva dos compostos sobre as células neoplásicas é uma das considerações fundamentais para obtenção de uma quimioterapia favorável para o paciente. Quanto menor a toxicidade em tecidos normais, maior o índice terapêutico favorável9. Há uma procura por moléculas que atuam em alvos específicos e assim tenham a capacidade de induzir seletivamente citotoxicidade em células tumorais por atuarem em aberrações que garantem a perpetuação do tumor e não atingem tecidos normais do paciente por não possuírem esses alvos ${ }^{16}$. Na literatura, o ácido betulínico é descrito como uma substancia que provavelmente possui citotoxicidade seletiva contra uma variedade de tipos específicos de neoplasias com ausência de efeitos colaterais sistêmicos².

Para avaliar os compostos estudados neste artigo quanto a essa característica, os derivados DAB2 e DAB3 tiveram sua seletividade testada em fibroblasto. Os resultados do presente estudo apontam que esses compostos não têm atividade que afete significativamente os fibroblastos in vitro, pois não houve alteração da viabilidade destas células. Assim, sugere-se que os compostos DAB2 e DAB3 possuem uma ação antineoplásica in vitro satisfatória contra células de câncer de adrenal com preservação da viabilidade de células hígidas. Novamente, os dados de Baratto apontam a mesma tendência para esses derivados do AB. No estudo deste autor, essas substâncias foram menos tóxicas sobre fibroblastos humanos em comparação com as linhagens tumorais testadas de ovário, mama, melanoma, tireoide e pulmão, com exceção do derivado DAB2 sobre as linhagens de tireoide e pulmão. 0 derivado mais ativo, DAB3, mostrou-se de três a cinco vezes mais tóxico nas linhagens cancerígenas testadas enquanto o DAB2 mostrou-se uma a três vezes mais seletivo para as linhagens celulares de ovário, melanoma e mama13.

0 tratamento de primeira linha para o câncer de adrenal, que não seja cirúrgico, oferecido no momento é a quimioterapia. Pode ser utilizada como tratamento 
adjuvante a cirurgia ou isoladamente em casos em que o câncer tem ressecção incompleta ou contraindicada e parece aumentar a sobrevida do paciente 20,210 mitotano é o único medicamento aprovado para o tratamento desta neoplasia, mesmo tendo poucas evidencias de sua eficácia ainda é o medicamento mais usada para este fim ${ }^{22}$. Este composto age propiciando alteração na função mitocondrial e no metabolismo de cortisol extra-adrenal, bloqueia esteroides e age como agente adrenolitico15. Infelizmente, o mitotano não é homogeneamente eficaz em todos os casos de carcinoma adrenocortical23. Assim, a quimioterapia disponível não tem uma eficácia ideal para lidar com esse câncer letal20. Essa medicação acomete uma alta parcela de pacientes com efeitos colaterais dosedependentes, principalmente gastrointestinais e neurológicos, provocando vômitos, diarreia e dor abdominal. Outras reações, menos comuns, ao medicamento são sonolência, letargia, depressão e vertigem ${ }^{15,24}$.

Não tem sido limitado à monoterapia, o potencial do ácido betulínico. Pode ser usado em associação com outros agentes quimioterápicos para sensibilizar as células a apoptose induzida por estes outros fármacos. Além de aumentar a eficácia destes medicamentos, diminui a ocorrência de efeitos colaterais e tóxicos destes por permitir a diminuição das doses administradas necessárias ${ }^{16}$. Foi encontrado um efeito de cooperação entre o ácido betulínico e fármacos anticancerígenos citotóxicos com diferentes modos de ação, como VP16, doxorrubicina, cisplatina, taxol, ou actinomicina D. Vale ressaltar que o tratamento de combinação com o ácido betulínico e outras drogas antitumorais colaborou para induzir a apoptose em diferentes linhagens celulares de tumores incluindo células p53 mutantes ${ }^{10}$.

\section{CONCLUSÃO}

Os dados presentes neste estudo sugerem que os derivados DAB2 e DAB3 tenham potencial para desenvolvimento de uma droga citotóxica para o CCA com seletividade favorável para uso terapêutico tanto isolados como concomitantes a outras drogas. Mas, a avaliação do ácido betulínico e seus derivados ainda está em fase préclínica de investigação. Para justificar sua progressão para fase clínica, ainda será necessário demonstrar a relevância das informações produzidas e antecipar os riscos de sua utilização25. Pelos resultados favoráveis encontrados neste estudo, a letalidade deste tipo de tumor e a falta de quimioterapia eficaz, sugere-se a consolidação dos dados obtidos com novos dados experimentais in vitro, seguidos por experimentos em modelos murinos.

\section{CONFLITOS DE INTERESSE}

Não houve conflitos de interesse.

\section{REFERÊNCIAS}

1. Instituto Nacional de Câncer, Rede de Atenção Oncológica [homepage na Internet]. Câncer na Crianca e no Adolescente no Brasil. [ Acesso em 9 de dezembro de 2013]. Disponível em: http://www.redecancer.org.br/.

2. Silva JN. Atividade antioxidante e citotóxica de extratos de plantas do semiárido brasileiro com potencial para desenvolvimento de fitoterápicos. Dissertação [Mestrado em Ciências Farmacêuticas] Universidade Federal do Piauí. 2015.

3. Ribeiro RC, Sandrini F, Figueiredo B, Zambettig GP, Michalkiewicz E, Laffertyi AR, et al. An inherited p53 mutation that contributes in a tissue-specific manner to pediatric adrenalcortical carcinoma. PNAS 2001; 98:9330-9335.

4. Michalkiewicz E, Sandrini R, Figueiredo B, Miranda ECM, Caran E, Oliveira-Filho AG, et al. Clinical and Outcome Characteristics of Children With Adrenocortical Tumors: A Report From the International Pediatric Adrenocortical Tumor Registry. J. Clin. Oncol. $2004 ; 22(5): 838-845$.

5. Figueiredo BC, Sandrini R, Zambetti GP, Pereira RM, Cheng $\mathrm{C}$, Liu W, et al. Penetrance of adrenocortical tumours associated with the germlineTP53R337H mutation. J Med Genet 2006; 43:91-96.

6. Moreira SG, Pow-Sang JM. Evaluation and Management of Adrenal Masses. Cancer Control 2002; 09:326-334.

7. Ray-Coquard I, Ghesquiere H, Bachelot T, Borg C, Biron $\mathrm{P}$, Sebban C, et al. Identification of patients at risk for early death after convential chemotherapy in solid tumours and lymphomas. Br J Cancer. 2001; 85:81622.

8. Mans DR, Rocha AB, Schwartsmann G. Anti-Cancer Drug Discovery and Development in Brazil: Targeted Plant Collection as a Rational Strategy to Acquire Candidate Anti-Cancer Compounds. Oncologist 2000; 5:185-198.

9. Baratto LC, Porsani MV, Pimentel IC, Netto ABP, Paschke R, Oliveira BH. Preparation of betulinic acid derivatives by chemical and biotransformation methods and determination of cytotoxicity against selected cancer cell lines. Eur. J. Med. Chem 2013; 68:121-131.

10. Fulda S, Debatin KM. Sensitization for Anticancer Drug-Induced Apoptosis by Betulinic Acid. Neoplasia 2005; 7:162-170.

11. Tan Y, Yu R, Pezzuto JM. Betulinic Acid-induced Programmed Cell Death in Human Melanoma Cells Involves Mitogen-activated Protein Kinase Activation. Clin. Cancer Res. 2003; 9:2866 -2875.

12. Shi W, Tang N, Yan WD. Synthesis and cytotoxicity of triterpenoids derive from betulin and betulinic acid via click chemistry. J Asian Nat Prod Res 2014; 16:1-11.

13. Baratto LC. Obtenção de derivados do ácido betulinico por meio de reações de biotransformação e semissíntese e avaliação das atividades antitumoral, antimicrobiana, antimalárica e anti-HIV. Tese 
[Doutorado em Ciências Farmacêuticas] Universidade Federal do Paraná; 2003.

14. Fassnacht $M$, Allolio B. Clinical management of adrenocortical carcinoma. Best Pract Res Clin Endocrinol Metab 2009; 23:273-289.

15. Ng L, Libertino JM. Adrenocortical Carcinoma: diagnosis, evaluation and treatment. J Urol 2003. $169: 5-11$.

16. Silva FS. Investigação de atividade antineoplasica de substancias puras isoladas de plantas do semiárido brasileiro e derivados sintéticos. Dissertação [Mestrado em Biotecnologia] - Universidade Estadual de Feira de Santana. 2007.

17. Chintharlapalli S, Papineni S, Lei P, Pathi S. Betulinic acid inhibits colon cancer cell and tumor growth and induces proteasomedependent and -independent downregulation of specificity proteins (Sp) transcription factors. BMC Cancer 2011; 11:371-383.

18. Chintharlapalli S, Papineno S, Ramaiah SK, Safe S. Betulinic Acid Inhibits Prostate Cancer Growth through Inhibition of Specificity Protein Transcription Factors. Cancer Res 2007; 67(6):2816-2823.

19. Costa-Lotufo LV, Montenegro RC, Alves APNN, Madeira SVF, Pessoa C, Moraes MEA et al. A Contribuição dos Produtos Naturais como Fonte de Novos Fármacos Anticâncer: Estudos no Laboratório Nacional de Oncologia Experimental da Universidade Federal do Ceará. Rev. Virtual Quim.2010; 2:47-58.

20. Maluf DF, Oliveira BH, Lalli E. Therapy of adrenocortical cancer: present and future. Am J Cancer Res 2011; 1(2):222-232

21. Terzolo M, Angeli A, Fassnacht M, Daffara F, Tauchmanova L , Conton PA, et al. Adjuvant Mitotane Treatment for Adrenocortical Carcinoma. N. Engl. J. Med. 2007; 356:2372-80.

22. Fassnacht M, Terzolo M, Allolio B, Baudin E, Haak H, Berruti A, et al. Combination Chemotherapy in Advanced Adrenocortical Carcinoma. N Engl J Med 2012; 366:2189-2197.

23. Kasperlik-Zaluska AA. Clinical results of the use of mitotane for adrenocortical carcinoma. Braz J Med Biol Res 2000; 33:1191-1196.

24. Ribeiro RC, Michalkiewicz E, Figueiredo BC, De Lacerda $L$,Sandrini $F$, Pianovsky MD, et al. Adrenocortical tumors in children. Braz J Med Biol Res 2000; 33: 1225-1234.

25. Goldim JR. A Avaliação ética da investigação científica de novas drogas: A importância da caracterização adequada das fases da pesquisa. Clinical \& Biomedical Research 2007; 27(1):66-73. 